\title{
Alto rendimiento clínico entre albuminuria semicuantitativa y proteinuria de 24 horas en pacientes con sospecha de síndrome hipertensivo del embarazo
}

\author{
Joaquín Errázuriz $V_{i}^{1}$, Álvaro Carrasco E. ${ }^{1}$, Fernando Ferrer $M^{1}{ }^{1}$, Álvaro Insunza $F^{1}$, \\ Ricardo Gómez M. ${ }^{1}$ \\ 1 Departamento de Ginecología, Unidad de Gestión Clínica de la Mujer y el Recién Nacido, Hospital Padre Hurtado, \\ Facultad de Medicina Clínica Alemana-Universidad del Desarrollo, Santiago. Chile.
}

\section{RESUMEN}

Antecedentes: La evaluación precisa de la proteinuria constituye un pilar importante para el diagnóstico del síndrome hipertensivo del embarazo (SHE). El estándar dorado para esta medición es la recolección de orina en 24 horas, pero debido a la duración de la toma de la muestra, alternativas como la albuminuria semicuantitativa se utiliza con mayor frecuencia en los servicios de urgencia de nuestro país. Objetivo: Evaluar el rendimiento diagnóstico de la albuminuria semicuantitativa y su asociación con proteinuria de 24 horas en pacientes con SHE. Métodos: Estudio retrospectivo de 145 pacientes con sospecha de SHE atendidas en el Hospital Padre Hurtado, Chile. A todas las pacientes se le realizó albuminuria semicuantitativa (categorizada entre $0+$ y $4+$ ) y proteinuria de 24 horas (positivo si $>0,3$ gramos/24 horas). Se realizó análisis por grupos compuestos de albuminuria semicuantitativa y resultado positivo en proteinuria de 24 horas. Resultados: Se evidenció una sensibilidad de $50 \%$, especificidad de $100 \%$, VPP de $100 \%$, VPN de $65,7 \%$, LR+ de 50 y un LR- de 0,5. Conclusión: La albuminuria semicuantitativa $\geq 2+$ muestra una fuerte asociación con proteinuria $\geq 0,3 \mathrm{~g} / 24$ horas y es un método rápido para evaluar SHE.

\section{PALABRAS CLAVE: Síndrome hipertensivo del embarazo, albuminuria semicuantitativa, proteinuria de 24 horas}

\section{SUMMARY}

Background: One of the basis for the diagnosis of pregnancy induced hypertension syndrome (PIHS), includes the precise evaluation of proteinuria. The gold standard for its evaluation is the collection of a 24-hour urine specimen, but because it is a slow method, other alternatives, such as semi-quantitative albuminuria have been used more frequently on our emergency rooms. Objective: To assess the diagnostic performance of semi-quantitative albuminuria and its association with proteinuria measured in a 24-hour urine specimen collection, in patients with PIHS. Methods: Retrospective study of 145 patients with clinical suspicion of PIHS who assisted to Hospital Padre Hurtado, Chile. Semi-quantitative albuminuria (categorized as 0 to $4+)$ and proteinuria measured in a 24-hour urine specimen collection was measured on every patient. Abnormal values of proteinuria were considered when values exceeded $0.3 \mathrm{~g} / 24$ hours. Composite outcomes analysis was done between albuminuria groups and positive proteinuria in $24 \mathrm{hrs}$. Results: Sensibility and specificity of semi-quantitative albuminuria was of $50 \%$ and $100 \%$, respectively, with a PPV: $100 \%$, NPV: $65.7 \%$, LR+: 50 and a LR-: 0.5 . Conclusion: semi-quantitative albuminuria $\geq 2+$ shows a strong association with proteinuria $\geq 0.3 \mathrm{~g} / 24$ hours and it could be used as a fast method to assess PIHS.

KEY WORDS: Pregnancy induced hypertension syndrome, semi-quantitative albuminuria, 24-hour urine proteinuria collection 


\section{INTRODUCCIÓN}

El síndrome hipertensivo del embarazo (SHE) es una de las principales causas de muerte materna y morbimortalidad fetal en todo el mundo. La Organización Mundial de la Salud (OMS) estima que existen anualmente más de 166 mil muertes por preeclampsia y su incidencia es de 5 a $10 \%$ (1). En Chile, su prevalencia varía entre el 7 y $10 \%$, siendo la primera causa de muerte materna desde 1990. En el período 1990-2000 hubo 181 muertes, con un promedio de 16,5 muertes/año, mientras que en el período 2000-2004 hubo 52 fallecimientos con un promedio de 10,4 muertes/año y de ellos, 34 fueron por eclampsia $(65,4 \%)$ (2). Adicionalmente, esta patología genera una alta tasa de complicaciones neonatales dadas por parto de pretérmino, restricción del crecimiento y muerte neonatal $(3,4)$.

El enfoque clínico de la paciente está dirigido a evaluar la severidad de la patología, realizar el diagnóstico diferencial entre los distintos tipos de hipertensión del embarazo y precisar la magnitud del daño causado por la hipertensión arterial (HTA) en los parénquimas maternos. Dentro de este contexto, la evaluación precisa de la proteinuria constituye un pilar importante para el diagnóstico del SHE.

Debido a que este síndrome constituye un amplio espectro de patologías con una significativa morbi-mortalidad, se requiere un diagnóstico preciso y rápido para así poder ofrecer el manejo adecuado. El estándar dorado para la medición de la proteinuria es la recolección de orina en 24 horas $(5,6,7)$. Sin embargo, esta prueba genera múltiples inconvenientes tanto para la paciente por el tiempo requerido para la recolección, como para las instituciones, ya que aumenta la estancia hospitalaria y se suma la recolección parcial de la muestra lo que retrasa el diagnóstico y la toma de decisiones. Debido a esto, se han planteado alternativas tales como tiras urinarias reactivas, recolecciones de orina en periodos más cortos, relaciones urinarias Proteína/Creatinina y la albuminuria semicuantitativa presente en la orina en pacientes con sospecha de SHE $(8,9,10)$.

La albuminuria semicuantitativa es posiblemente uno de los test más utilizados en las urgencias de nuestro país. Esta prueba consiste en utilizar ácido sulfosalicílico al 30\%. Este reactivo químico es capaz de producir la precipitación de proteínas a través de la acidificación de la orina. Los resultados positivos se pueden interpretar como un posible indicio de complicaciones hipertensivas en el embarazo, infección del tracto urinario o enfermedad renal crónica (11). A pesar de las implicancias tanto en el diagnóstico como pronóstico del embarazo, su cuantificación y asociación con las complicaciones del SHE son desconocidas. El número de estudios realizados hasta la fecha son escasos y no permiten concluir con real certeza la asociación que existe entre sus valores y la proteinuria de 24 horas.

De acuerdo a este planteamiento, el objetivo de este estudio fue determinar el rendimiento diagnóstico de la albuminuria semicuantitativa y su asociación con proteinuria de 24 horas en pacientes con sospecha de SHE.

\section{PACIENTES Y MÉTODOS}

Se realizó un estudio retrospectivo, en el que se utilizó información almacenada en la base electrónica de ingresos de urgencias y posteriormente en las fichas respectivas de la unidad de Obstetricia del Hospital Padre Hurtado, Santiago, Chile. Se incluyeron a todas las pacientes con embarazo mayor a 20 semanas que fueron hospitalizadas por sospecha de SHE, durante el período comprendido entre Enero y Diciembre de 2011. Se registraron características demográficas de la población en estudio (edad, paridad, edad gestacional, peso recién nacido, vía del parto). Se extrajeron de la base de datos electrónica de urgencia ginecológica los resultados de la prueba de albuminuria semicuantitativa tomados al ingreso, en pacientes con cifras tensionales $\geq 140 / 90 \mathrm{mmHg}$ en 2 determinaciones, clínica de SHE (tinnitus, fotopsia o epigastralgia), o con diagnóstico previo de hipertensión crónica (HTAc).

El test de albuminuria semicuantitativa se realizó tomando una alícuota de orina de $5 \mathrm{ml}$ que se mezcló con tres gotas del ácido sulfosalicílico al $30 \%$, creándose un gradiente de turbidez. Los hallazgos se clasificaron como sigue: Negativa: sin turbidez; 1+: hay turbidez no granular; 2+: hay turbidez granular; $3+$ : turbidez considerable con aglutinación; 4+: turbidez densa con masa granular aglutinada de gran tamaño que puede solidificarse (12) (Figura 1). Esta interpretación fue realizada por la matrona de turno. Se excluyeron a pacientes en trabajo de parto, sangrado vaginal, pacientes con disfunción renal, parto con menos de 24 horas después del ingreso, no realización de sedimento urinario al ingreso, o no recolección de orina de 24 horas por normalización de la presión arterial en su hospitalización.

Se revisaron las fichas de todas las pacientes hospitalizadas con sospecha de SHE con las características descritas y que presentaron cifras tensionales elevadas a las 6 horas de su hospitalización, con indicación de proteinuria de 24 horas. Esto consistió en solicitar a la paciente recolectar orina durante ese período y su posterior cuantificación de proteínas en laboratorio. Se consideró proteinuria anormal cuando el valor fue $\geq 0,3$ gramos en 24 horas. 


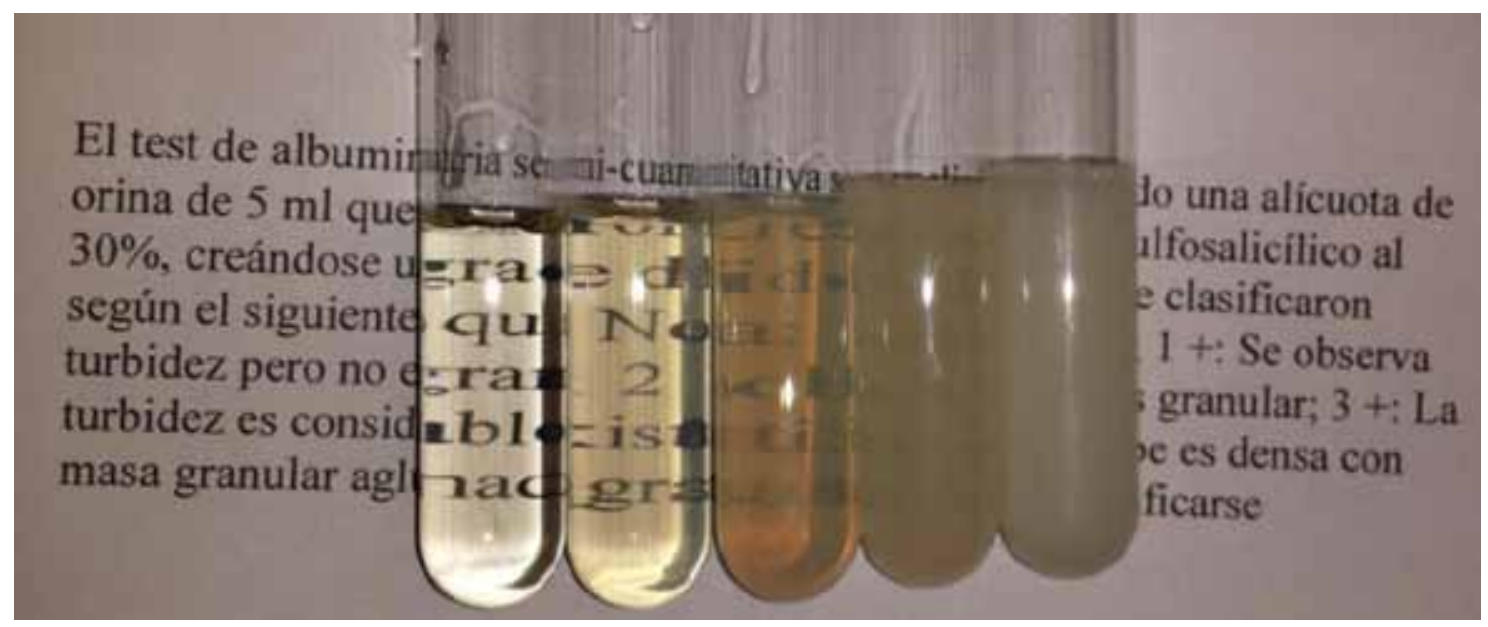

Figura 1. Ejemplo de albuminuria semi-cuantitativa. De izquierda a derecha: albuminuria negativa $(0+), 1+$, $2+, 3+, 4+$.

Toda la información se descargó en una base de datos Microsoft Excel 2000. Se calcularon los valores de Sensibilidad, Especificidad, Valor predictivo positivo (VPP) y negativo (VPN); Likelihood Ratio positivo (LR+) y negativo (LR-). Las comparaciones univariadas de datos dicotómicos se realizaron con el Test Exacto de Fisher. Las comparaciones entre grupos se realizaron con el test de Kruskal - Wallis para datos no paramétricos. El análisis post - hoc de comparaciones por pares para esta prueba fue realizado usando el procedimiento de Dunn con corrección de Bonferroni para comparaciones múltiples. Las correlaciones fueron realizadas usando Rho de Spearman para datos no paramétricos. Se consideró diferencia significativa un valor $p<0,05$ (dos colas). Los datos fueron analizados con el software estadístico SPSS (v 22.0).

\section{RESULTADOS}

Durante el período estudiado, hubo un total de 145 pacientes hospitalizadas por sospecha de SHE con embarazo mayor a 20 semanas. Las características demográficas se describen en la Tabla I. Los elementos clínicos sugerentes de SHE más frecuentes al ingreso fueron: cifras tensionales elevadas ( $\geq 140 / 90 \mathrm{mmHg}$ ) (93 casos; $64 \%$ ), clínica de SHE asociado a albuminuria semicuantitativa (+) (34 casos; 23\%), o con diagnóstico previo de HTAc (18 casos; $13 \%$ ).

Los hallazgos del test de albuminuria semicuantitativa tomado al ingreso, clasificados en cruces fueron: negativo $(0+)$ en $40(27,6 \%) ; 1+$ en 68 $(46,9 \%) ; 2+$ en $14(9,7 \%) ; 3+$ en $12(8,3 \%)$ y $4+$ en $11(7,6 \%)$ pacientes, respectivamente (Tabla II).

Para evaluar asociación entre albuminuria semicuantitativa y proteinuria en 24 horas se dividieron los resultados en 2 grupos, 0 - 1+ y 2 4+. Al realizar el análisis de rendimiento del test diagnóstico se evidenció una sensibilidad de $50 \%$, especificidad de 100\%, VPP de 100\%, VPN de $65,7 \%$, LR+ (no calculable), y un LR- de 0,50. La asociación compuesta $(0-1+, 2-4+)$ evaluada con Test Exacto de Fisher fue 47,66 ( $p<0,001)$. No se evidenciaron casos de proteinuria $\geq 0,3$ gramos en 24 horas con albuminuria semicuantitativa negativa (Tabla III).

\section{Tabla I \\ CARACTERÍSTICAS DEMOGRÁFICAS DE LA POBLACIÓN ESTUDIADA Y RESOLUCIÓN DEL PARTO}

Características de la población en estudio

Edad (años)

$26,3 \pm 7,53$

Paridad

Rango: 0-7

Edad gestacional al ingreso

$31 \pm 6 \mathrm{~s}$

Peso recién nacido

$3.289 \pm 675 \mathrm{~g}$

Clínica de ingreso:

$\mathrm{PA} \geq 140 / 90 \mathrm{mmHg}$

$93(64 \%)$

Clínica de SHE+Albuminuria (+)

$34(23 \%)$

Hipertensión arterial crónica

$18(13 \%)$

Vía de parto:

Vaginal

$98(67,1 \%)$

Fórceps

$10(6,8 \%)$

Cesárea

$38(26,1 \%)$ 
Posteriormente se realizó la prueba de KruskalWallis para determinar diferencias en los resultados de proteinuria de 24 horas entre los grupos que diferían en su albuminuria semicuantitativa: negativa $(0+)(n=40), 1+(n=68), 2+(n=14), 3+(n=12)$ y $4+(n=11)$. Las distribuciones de las proteinurias de 24 horas no fueron similares para todos los grupos, según la inspección visual de un diagrama de cajas. Las proteinurias de 24 horas fueron significativamente diferentes entre los distintos niveles de albuminuria semicuantitativa, $x^{2}(3 \mathrm{GL})=110,208$; $p<0,001$. El análisis post hoc reveló diferencias estadísticamente significativas en las proteinurias de 24 horas entre los grupos de albuminuria semicuantitativa: Negativa $(0+)$ y $1+(p<0,001)$, Negativa $(0+)$ y $2+(p<0,001)$, Negativa $(0+)$ y $3+(p<0,001)$, Negativa $(0+)$ y $4+(p<0,001) ; 1+$ y $2+(p<0,001), 1+$ y $3+(p<0,001), 1+y 4+(p<0,001)$, no hubo diferencias en cualquier otra combinación de grupos.

Al realizar la prueba de correlación de Spearman para distribuciones no paramétricas entre las proteinurias de 24 horas y la albuminuria semicuantitativa se observó una correlación positiva muy alta, $\operatorname{Rho}_{S}(143)=0,869(p<0,001)$.

\section{Tabla II \\ DISTRIBUCIÓN DE LA ALBUMINURIA SEMICUANTITATIVA EN FUNCIÓN DE LA PROTEINURIA DE 24 HORAS}

\begin{tabular}{cccc}
\hline $\begin{array}{c}\text { Albuminuria } \\
\text { semicuantitativa }\end{array}$ & $\begin{array}{c}\text { Proteinuria } \\
<0,3 \mathrm{~g} / 24 \mathrm{~h}\end{array}$ & $\begin{array}{c}\text { Proteinuria } \\
\geq 0,3 \mathrm{~g} / 24 \mathrm{~h}\end{array}$ & Total \\
\hline $0+$ & 40 & 0 & 40 \\
$1+$ & 31 & 37 & 68 \\
$2+$ & 0 & 14 & 14 \\
$3+$ & 0 & 12 & 12 \\
$4+$ & 0 & 11 & 11 \\
\hline Total & 71 & 74 & 145 \\
\hline
\end{tabular}

\section{DISCUSIÓN}

Nuestros resultados muestran una correlación positiva muy alta entre la albuminuria semicuantitativa y la proteinuria de 24 horas. El análisis de asociación resulta positivo y altamente significativo con albuminuria mayor o igual $2+$, con una especificidad y VPP que permiten tomar una conducta clínica precoz con baja posibilidad de error. Es importante señalar que el valor de LR+ no es calculable dada la especificidad obtenida de 100\% (división por cero), posiblemente relativa en función del $\mathrm{n}$ de la muestra.

Es importante destacar además que con un resultado de albuminuria semicuantitativa negativo no se encontraron casos de proteinuria de 24 horas mayor a 0,3 gramos, lo que permite, según nuestros resultados, excluir el diagnóstico de preeclampsia. Las comparaciones entre grupos confirman estos resultados y pueden interpretarse como que un resultado negativo $(0+)$ tiene un $100 \%$ de rendimiento para excluir una preeclampsia, un resultado mayor a $2+$ tiene un rendimiento de $100 \%$ para detectar el diagnóstico y con un resultado de 1+ solamente se predicen un $54 \%$ de las preeclampsias, no existiendo diferencias significativas para el diagnóstico cuando la albuminuria es mayor a $2+$. Proponemos un esquema de manejo según resultados de proteinuria semicuantitativa (Figura 2).

Nuestros resultados configuran a la albuminuria semicuantitativa como un test diagnóstico de excelente rendimiento, permitiendo afirmar que se podría sustituir una prueba por otra con la ventaja de ser mucho menos complicada en la obtención de la información y que además está disponible de forma casi inmediata. No se encontró en la bibliografía estudios que permitan comparar conclusiones, sin embargo existe evidencia que muestra en embarazadas una gran variabilidad en la excreción de proteínas durante el día, por lo cual cualquier estudio de SHE menor a 24 horas no sería representativo (13).

Tabla III

CATEGORIZACIÓN COMPUESTA DE ALBUMINURIA 24 HRS Y ANÁLISIS DE EFICACIA DIAGNÓSTICA

\begin{tabular}{cccc}
\hline Albuminuria SC & Proteinuria $\geq 0,3 \mathrm{~g} / 24 \mathrm{~h}$ & Proteinuria $<0,3 \mathrm{~g} / 24 \mathrm{~h}$ & \multicolumn{1}{c}{ Total } \\
\hline $2-4+$ & $37(\mathrm{~A})$ & 0 (C) & $37(\mathrm{~A}+\mathrm{C})$ \\
$0-1+$ & $37(\mathrm{~B})$ & $71(\mathrm{D})$ & $108(\mathrm{~B}+\mathrm{D})$ \\
\hline Total & $74(\mathrm{~A}+\mathrm{B})$ & $71(\mathrm{C}+\mathrm{D})$ & 145 \\
\hline
\end{tabular}

Sensibilidad: $A /(A+B)=37 / 74=0,5(50 \%)$. Especificidad: $D /(C+D)=71 / 71=1(100 \%)$. VPP: $A(A+C)=37 / 37=1(100 \%)$. VPN: $D / B+D=71 / 108=0,657(65,7 \%)$. LR+: Sensibilidad/(1-Especificidad) $=0,5 /(1-1)=$ No calculable (división por cero). LR-: (1-Sensibilidad)/Especificidad $=(1-0,5) / 1=0,50$. Test Exacto de Fisher: 47,66 $p<0,001$. 

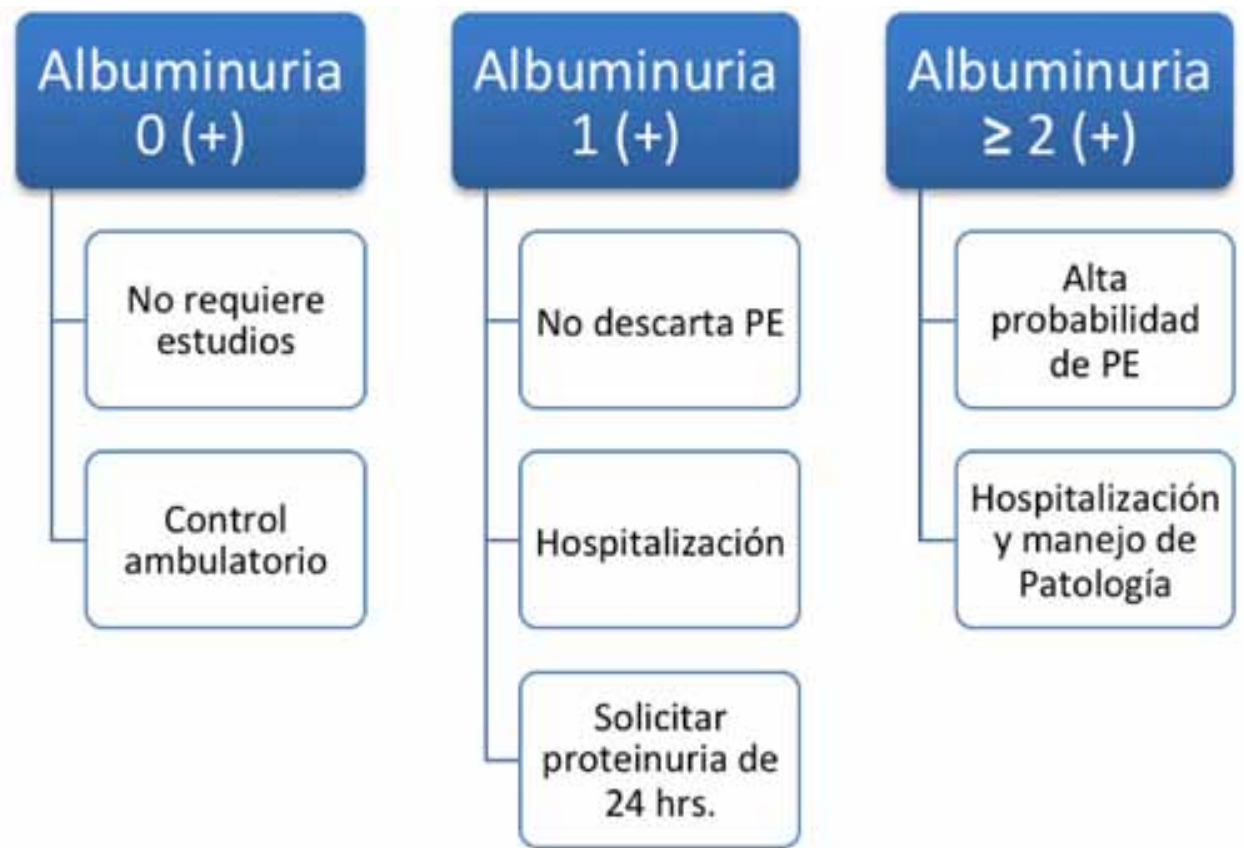

Figura 2. Esquema de manejo según albuminuria semicuantitativa.

Un elemento a considerar es la concordancia diagnóstica inter-observador. Al ser un test semicuantitativo en su esencia, la categorización final puede ser diferente según la experiencia y entrenamiento de quien realiza el test, pudiendo constituir un sesgo importante en este estudio. Se requiere investigación adicional para determinar la homologación de las mediciones en la detección de proteinuria. Ahora el énfasis debe ser mejorar las técnicas de diagnóstico de manera que podamos confirmar la presencia o ausencia de proteinuria superior a un umbral de 0,3 $\mathrm{g} / 24$ horas en cualquier entorno clínico, preferiblemente, con una prueba tan simple como albuminuria semicuantitativa. Si esto se hace de forma fiable, la necesidad de una confirmación a través de la proteinuria de 24 horas para un diagnóstico de preeclampsia pudiese convertirse en una cosa del pasado. Los hallazgos de este estudio podrían permitirnos orientar protocolos de manejo y optimizar la administración de los recursos disponibles en ésta y otras instituciones similares del país.

\section{CONCLUSIÓN}

La albuminuria semicuantitativa es un método barato y simple que muestra una asociación significativa con proteinuria $\geq 0,3 \mathrm{~g} / 24$ horas cuando el resultado es $\geq 2+$ y que permitiría descartar el diagnóstico de preeclampsia si la albuminuria resulta negativa. Este resultado permitiría agilizar el manejo de las pacientes con SHE en quienes se sospecha preeclampsia, modificando la conducta clínica, muchas veces retrasada por el estándar dorado actual como es la albuminuria de 24 horas.

\section{REFERENCIAS}

1. World Health Organization International Collaborative Study of Hypertensive Disorders of Pregnancy. Geographic variation in the incidence of hypertension in pregnancy Am J Obstet Gynecol 1998;158:80-3.

2. Donoso Siña E. Reducción de la mortalidad materna en Chile de 1990 a 2000. Rev Panam Salud Pública 2004;15:326-30.

3. Xiong X, Demianczuk NN, Saunders LD, et al. Impact of preeclampsia and gestational hypertension on birth weight by gestational age. Am J Epidemiol 2002;155:203-9.

4. Eskild A, Romundstad PR, Vatten LJ. Placental weight and birth weight: does the association differ between pregnancies with and without preeclampsia? Am J Obstet Gynecol 2009;201:595.e1-e5.

5. Higby K, Suiter CR, Phelps JY, et al. Normal values of urinary albumin and total protein excretion during pregnancy. Am J Obstet Gynecol 1994;171:984-9. 
6. Côté AM, Firoz T, Mattman A, et al. The 24-hour urine collection: gold standard or historical practice? Am J Obstet Gynecol 2008;199:625.e1-6.

7. Durnwald C, Mercer B. A prospective comparison of total protein/creatinine ratio versus 24-hour urine protein in women with suspected preeclampsia. Am J Obstet Gynecol 2003;189:848-52.

8. McElderry LM, Tarbit IF, Cassells-Smith AJ. Six methods for urinary protein compared. Clin Chem 1982;28:356-60.

9. Gangaram R, Ojwang PJ, Moodley J, Maharaj D. The accuracy of urine dipsticks as a screening test for proteinuria in hypertensive disorders of pregnancy. Hypertens Pregnancy 2005;24:117-23.
10. Rodriguez-Thompson D, Lieberman ES. Use of a random urinary protein-to-creatinine ratio for the diagnosis of significant proteinuria during pregnancy. Am J Obstet Gynecol 2001; 185:808-11.

11. Airoldi, J. Weinstein L. Clinical Significance of proteinuria in pregnancy. Obstet Gynecol Survey 2007;62:11724.

12. Zweig M H, Campbell G. Receiver-Operating Characteristic (ROC) Plots: A fundamental evaluation tool in clinical medicine. Clin Chem 1993;39:561-77.

13. Lindow SW, Davey DA. The variability of urinary protein and creatinine excretion in patients with gestational proteinuric hypertension. Br J Obstet Gynaecol 1992;99:869-72. 